\title{
Balkanologie
}

Balkanologie Revue d'études pluridisciplinaires

Vol. X, $n^{\circ} 1-2 \mid 2008$

Volume $X$ Numéro 1-2

\section{Le Danube dans les Balkans : introduction au dossier}

Emmanuelle Boulineau

\section{OpenEdition}

Journals

Édition électronique

URL : http://journals.openedition.org/balkanologie/382

DOI : $10.4000 /$ balkanologie.382

ISSN : 1965-0582

Éditeur

Association française d'études sur les Balkans (Afebalk)

Référence électronique

Emmanuelle Boulineau, « Le Danube dans les Balkans : introduction au dossier », Balkanologie [En ligne], Vol. X, nº 1-2 | 2008, mis en ligne le 03 juin 2008, consulté le 17 décembre 2020. URL : http:// journals.openedition.org/balkanologie/382 ; DOI : https://doi.org/10.4000/balkanologie.382

Ce document a été généré automatiquement le 17 décembre 2020.

(c) Tous droits réservés 


\title{
Le Danube dans les Balkans : introduction au dossier
}

\author{
Emmanuelle Boulineau
}

1 Ce dossier sur le Danube dans les Balkans souhaite contribuer à la réflexion sur la place $\mathrm{du}$ fleuve dans la péninsule balkanique, dans l'esprit interdisciplinaire porté par la revue Balkanologie. Le choix de la thématique a été guidé par un premier constat sur la rareté des études sur le fleuve dans son cours aval. Certes, les conflits de la décennie 1990 et les pollutions répétées ont placé le Danube au cœur de l'actualité, mais les études scientifiques sont plutôt rares. Le Danube et les écrits qu'il a suscités semblent plutôt s'arrêter à Budapest, Vienne ou tout au plus à Belgrade à la limite de cette Mitteleuropa dont il est un symbole. C'est oublier - ou du moins sous-estimer - la portée de ce fleuve comme trait d'union entre l'Est et l'Ouest du continent européen, son importance historique comme porteur de la frontière du limes romain ou comme centre des tentatives de réorganisation de l'administration ottomane (vilayet du Danube) ou encore sa place dans les recompositions géopolitiques actuelles autour de la mer Noire dans laquelle il débouche.

2 Il est donc grand temps d'apporter une pierre à l'édifice de la connaissance du Danube dans les Balkans. Les 6 textes rassemblés ici relèvent de l'analyse géopolitique, de la géographie ou encore de la géohistoire. Ils résultent dans une large mesure des travaux de jeunes chercheurs, aménageurs, géographes, géopoliticiens au contact avec le terrain. Le regard de collègues étrangers a été aussi sollicité pour qu'ils expriment leur vision des enjeux danubiens.

3 Un premier thème ressort de ce dossier, celui de la perspective d'intégration des Balkans dans L'Europe via l'artère danubienne. Il est désormais impossible de penser la place du Danube dans les Balkans indépendamment de la position du bassin danubien en Europe. Seul grand fleuve européen qui par son orientation est-ouest permet de faire le lien entre l'Ouest et l'Est du continent, le Danube cristallise de nombreuses attentes quant à la recomposition du continent européen. De ce point de vue, l'éclairage de ce dossier sur la partie balkanique du fleuve fournit une réflexion sur les effets d'héritages balkaniques mais aussi sur les processus de différenciation à l'œuvre 
dans les Balkans pour cerner les vicissitudes d'une construction européenne qui achoppe régulièrement sur les Balkans (question du Kosovo, élargissement aux États issus de la Yougoslavie ou à la Turquie...). La lecture pessimiste revient sur les "questions balkaniques" à la charnière d'un Orient et d'un Occident; la lecture optimiste souligne la capacité des Balkans à être des catalyseurs de choix européens souvent inédits.

Dans le premier article du dossier, Jean-Marie Gauthey dresse un panorama des enjeux géopolitiques sur le Danube pour la construction d'une coopération internationale. Dans son analyse historique, il rappelle ainsi que le Danube a été l'objet d'une volonté précoce de gestion internationale, contrecarrée par l'histoire des $\mathrm{XIX}^{\mathrm{e}}$ et $\mathrm{XX}^{\mathrm{e}}$ siècles. Pour la période contemporaine l'auteur montre que la coopération s'apparente à une surimposition d'instances internationales davantage sectorielles que réellement tournées vers une intégration territoriale de ce vaste bassin fluvial à la dynamique politique et économique de l'Europe. Les recompositions géopolitiques dans la partie balkanique du fleuve constituent à cet égard un laboratoire intéressant de ces processus.

5 S'inscrivant lui aussi dans le questionnement sur l'intégration européenne du Danube balkanique, l'article de Veselin Siarov envisage une prospective territoriale avec l'outil du système d'information géographique (SIG) qui permet de croiser des informations qualitatives et quantitatives. La méthode expérimentale articule SIG et modèle prospectif de différenciation spatiale, afin de dégager des discontinuités spatiales et de raisonner sur la place de la partie aval du fleuve dans la dynamique générale du bassin danubien. L'analyse souligne le manque de connexion des Balkans à la dynamique d'ensemble et les lacunes d'une cohésion du bassin danubien encore à construire au niveau européen.

6 Centré sur la ville roumaine de Călăraşi et son rapport au Danube qui la traverse, la contribution d'Odile Bratosin montre comment la ville investit ou non le fleuve. L'intégration du Danube dans la dynamique européenne est envisagée ici du point de vue des politiques de développement régional appuyé sur l'émergence de villes-centres régionales. L'auteur explique ici le blocage des régions de développement et de l'eurorégion centrées toutes deux sur Călăraşi par le jeu des acteurs politiques qui contribuent à détourner la ville du fleuve. En revanche, la mémoire de cette ville danubienne est reprise en main par la société civile, un paradoxe entre acteurs institutionnels et populations riveraines intéressant à souligner dans les relations entre une ville et son fleuve.

7 Le second thème qui se dégage de ce dossier concerne les frontières sur le Danube dans les Balkans. L'interrogation sur le fleuve comme support de frontière taraude la réflexion sur les grands fleuves. Le Danube en est une formidable illustration: il traverse dix pays européens et porte notamment la plus longue frontière fluviale d'Europe entre la Roumanie et la Bulgarie. Ce dernier élément laisserait à penser que le Danube constitue une frontière stabilisée dans sa partie balkanique mais il n'en est rien. Le cours aval du fleuve est le lieu d'une dynamique nouvelle des frontières. La reconnaissance de nouveaux États transforme d'anciennes limites administratives en frontières internationales, c'est le cas du segment frontalier danubien entre la Croatie et la Serbie. L'élargissement de l'Union européenne augure une défonctionnalisation des frontières, introduisant une distinction entre ce qu'il est convenu d'appeler désormais les frontières internes (entre pays-membres) et les frontières externes de 
l'UE au contact des pays voisins. Les contributions proposent ici de décliner différentes formes que peut prendre la frontière sur le Danube dans la péninsule balkanique.

Maria Pătroescu et Radu Necşuliu envisagent la frontière internationale danubienne entre Roumanie et Serbie comme porteuse d'un territoire transfrontalier à construire dans le cadre de la réserve naturelle des Portes de Fer. La perspective sur la frontière danubienne passe ici d'une vision linéaire d'une frontière séparante à la construction en cours d'un territoire transfrontalier que la biosphère commune rassemble. Certes le risque d'instrumentalisation de la nature à des fins politiques est présent, mais les deux auteurs l'écartent en replaçant leur propos dans un questionnement environnemental sur la gestion d'un grand fleuve. Le rôle des institutions internationales est ainsi souligné et la comparaison avec d'autres projets de parcs transfrontaliers, filée à travers l'appareil bibliographique, montre l'importance d'un tel questionnement dans des pays dits en transition. La nécessité de reconstruire des liens entre la Serbie et la Roumanie passe ainsi par le fleuve.

9 Partant de la carte actuelle des frontières des États balkaniques, Emmanuelle Boulineau revient sur la question du Danube comme marqueur de frontière en étudiant les expertises frontalières en 1919-1920, au moment où la plupart de ces États apparaissent sur la carte d'Europe. Frontière linéaire, frontière naturelle, bonne et mauvaise frontière, ces notions sont discutées et rejetées par les experts géographes mandatés par les pays. Pour le démontrer, l'auteur replace la réflexion des géographes dans le cadre de Balkans traversés de fronts plus que de frontières, de discontinuités multiples sans recouvrement spatial. Elle montre par la comparaison entre le Rhin et le Danube à quel point les enjeux, à première vue communs à deux grands fleuves européens, divergent et que la frontière fluviale linéaire est trompeuse selon l'échelle à laquelle on l'observe.

10 Avec sa contribution sur les Lipovènes du delta du Danube, Frédéric Beaumont rappelle enfin l'importance des nombreuses minorités balkaniques en position de marche des constructions politiques nationales. "A la frontière ", c'est à dire en jouant de la marginalité à la fois religieuse et spatiale de cette communauté de Vieux-croyants, le cas des Lipovènes redonne toute son importance à l'enjeu minoritaire des communautés religieuses orthodoxes entre Balkans et Russie. Le delta du Danube a constitué un espace refuge comme ailleurs dans les Balkans les montagnes ont pu l'être. L'auteur montre ainsi comment ce peuplement marginal numériquement a su se maintenir par-delà les querelles géopolitiques du $\mathrm{XX}^{\mathrm{e}}$ siècle sur le delta du Danube, grâce notamment à ses privilèges économiques et à sa position de confins. "Territoire incertain ", le delta a accueilli flux et reflux de population au gré des changements de frontières et des politiques de colonisation des États retracés par l'auteur.

11 Ce dossier inaugure aussi une nouvelle ère pour la revue Balkanologie avec la publication en ligne qui permet d'accroître sa diffusion mais aussi de proposer des textes qui s'appuient sur des documents variés en couleur dont le web permet de rendre la qualité et la précision. Que la couleur du « beau Danube bleu » agrémente donc la lecture de ces travaux de recherche 


\section{AUTEUR}

\section{EMMANUELLE BOULINEAU}

Maître de conférenceUniversité de Lyon, ENS LSH, Géophile- UMR 5600 Environnement ville sociétéemmanuelle.boulineau@ens-lsh.fr 